\title{
Assessment of dose reduction and influence of gantry rotation time in CT abdomen examinations
}

\author{
M.K. Saeed ${ }^{*}$, N. Tammam², A. Sulieman 3 \\ ${ }^{1}$ Department of Radiological Sciences, Najran University, Najran, Saudi Arabia \\ 2Physics Department, College of Sciences, Princess Nourah Bint Abdulrahman University, Riyadh, Saudi Arabia \\ ${ }^{3}$ Radiology and Medical Imaging Department, Prince Sattam bin Abdulaziz University, Alkharj, Saudi Arabia
}

\section{- Original article}

\author{
*Corresponding authors: \\ Mohamedrick Saeed, PhD., \\ E-mail: \\ mohamedrick@gmail.com
}

Revised: March 2020

Accepted: June 2020

Int. J. Radiat. Res., January 2021; 19(1): 223-230

DOI: $10.29252 /$ ijrr.19.1.223

\begin{abstract}
Background: Worldwide the computed tomography (CT) scanning is recognized as a high radiation dose modality. This article aimed to estimate the radiation dose reduction and radiogenic risks for adult patients undertaking abdominal CT examinations. Materials and Methods: A total of 128 patients were studied using 2, 4, 16 and 64 slice CT scanners. The patients were divided into two categories: the first category as control category (80 patients), and the other as optimisation category (48 patients). The optimisation protocol was based on decreasing the gantry rotation time. Results: In general, the faster gantry rotation times $(0.7 \mathrm{~s} /$ rot and $0.5 \mathrm{~s} / \mathrm{rot}$ ) resulted in dose reduction while maintaining images noise within the acceptable range. After dose optimization, the overall cancer risk was reduced by $21 \%, 49 \%, 29 \%$, and $16 \%$, for the patients undergoing abdominal examinations on 2, 4, 16 and 64 slice CT scanners, respectively. Conclusion: The patient dose optimisation during CT abdomen was investigated. By lowering gantry rotation time, a radiation dose reduction of up to $30 \%$ was achieved without compromising the diagnostic findings. Accordingly, the attention of all technologists necessary to take advantage of the dose reduction methods reported in this study.
\end{abstract}

Keywords: Dose reduction, CT, virtual dose, abdomen.

\section{INTRODUCTION}

Since the advent of CT scanners, its utilizations started continues to grow substantially, however, concern about radiation risks from CT also increased. Worldwide the CT imaging is recognized as a high radiation dose modality and responsible for $70 \%$ medical radiation exposure (1). The mean of the effective dose values varies in literature according to the type of CT examinations. For example, the overall dose received by patients undergoing abdomen and pelvis examinations range between $8 \mathrm{mSv}$ and $14 \mathrm{mSv}{ }^{(2-4)}$. Due to the high radiation dose associated with $\mathrm{CT}$ imaging, its evaluation becomes an important issue. Furthermore, an attempt to reduce radiation dose is critically important.

The radiation output of a CT scanner and the stochastic health risk to the patient's body were commonly evaluated using computed tomographic dose index (CTDI) and effective dose, respectively. The CTDI allows us to estimate the amount of energy delivered to an acrylic phantom per gantry rotation. However, the effective dose takes into account the different sensitivity of tissues and organ, thus providing an initial estimation of radiogenic risk (5). Before the advent of helical scanners, the CTDI weighted (CTDIw) calculated by measuring the dose at the center and periphery of the phantom (16 cm and/or $32 \mathrm{~cm}$ diameter) using a pencil ionization chamber. The CTDIw concept was previously used for rotate-stationary, wide 
fan beam or narrow fan beam scanners (5). For helical scanners, however, the CTDIw concept breaks down because of pitch variation. Accordingly, the volume CTDI (CTDIvol) was introduced by the American Association of Physicists in Medicine (AAPM) (3). CTDIvol is also based on measurements obtained when scanning either a $16 \mathrm{~cm}$ or $32 \mathrm{~cm}$ phantom. However, CTDIvol calculated by dividing the CTDIw by pitch value (6). The dose-length product (DLP) is another quantity used to evaluate the CT tube radiation output based on the scan length and CTDIvol (5). These quantities provide specific information about proportional variation in dose according to modification in the parameters of CT examination.

The CT imaging of the abdomen is one of the most common radiological examinations, as it helps detect diseases of the colon and other internal organs. Moreover, it's used to evaluate potential malignancies. However, during CT abdomen procedure, kidney dose may be as much as 30-50 mGy, even though kidneys are not the target of imaging procedure (7). One technique in which the radiation dose to the radiosensitive organs can be reduced by 20$50 \%$ is the use of shielding (8). However, shields are associated with greater image noise and streak artefacts (8). Some studies have been published in radiation protection to patients during CT procedures (9-14). These studies have shown that there is a wide range of dose values and acquisition protocols. Furthermore, the data available on patient doses in CT procedures are generally outdated because of the continuous development of CT X-ray generators and technologic innovation that have taken place over the past decade from single-slice CT (15) to 640 slices (16). In previous literature, CT dose reduction achieved by using several techniques and strategies such as tube current (mAs), tube voltage $(\mathrm{kVp})$, scan length modulation, and iterative reconstruction algorithms (17). However, patients are still exposed to a higher amount of radiation in CT imaging compared with other imaging modalities

The modern CT scanners use rapid imaging technology to reduce radiation dose received by patients, such as fast gantry rotation times. Time reduction is a useful feature when imaging the abdomen region because it reduces the artefacts (18). However, streaking artefacts are also caused by decreasing gantry rotation time. Recently, Beeres et al. evaluated the dose reduction in chest CT examination based on accelerating gantry rotation time (18). This study aimed to evaluate the influence of rotation time on radiation exposure and image quality during abdomen examinations using several CT scanners. In addition, patients' radiogenic risks were also investigated.

\section{MATERIALS AND METHODS}

In Feb 2018, the study was ethically cleared by the Scientific Research Ethics Committee at Najran University (Registration number: 07-028-18EC). Informed consent was obtained from all individuals included in the study before the commencement of data collection. A total of 128 patients were divided into two categories: the first category (A) as control category (80 patients), and the other as optimisation category (48 patients). In addition, the patients were divided into four groups based on CT scanner type. All patients suffered from abdomen problems that required referring them to the CT department. The study was performed with four CT scanners. Table 1 gives a summary of the distribution of patients groups and the CT scanners. All examinations of the control category were performed with local abdomen protocols using $5.0 \mathrm{~mm}$ slice thickness with various imaging parameters based on patients' sizes and scanners' models. Other parameters used in these protocols such as pitch, $\mathrm{kVp}, \mathrm{mAs}$ and rotation time values were ranged between 0.8-4.5, $110-120 \mathrm{kVp}, 120-200 \mathrm{mAs}, 0.6-1.0$ seconds, respectively. All quality-control tests were implemented to the scanners by expert medical physicists before the collection of data. Technical parameters of the exposure and patients anthropometric data such as age, and weight were collected for all groups underwent abdomen CT scanning at the time of the examination. In this study, the dose optimisation strategy relied on reduction in gantry rotation

Int. J. Radiat. Res., Vol. 19 No. 1, January 2021 
times while maintaining image noise and artefact within an acceptable range. The rotation times were decreased by factors ranged between 1.2 and 1.4 as follows: group A 1.0, 0.7 s/rot; group B 0.7, 0.5 s/rot; group C $0.7,0.5$ s/rot; group D $0.6,0.5 \mathrm{~s} /$ rot.

Table 1. Distribution of patient groups according to the CT systems.

\begin{tabular}{|c|c|c|c|c|}
\hline \multirow{2}{*}{$\begin{array}{l}\text { Group } \\
\text { code }\end{array}$} & \multirow{2}{*}{$\begin{array}{c}\text { CT scanner } \\
\text { (Slices number) }\end{array}$} & \multirow{2}{*}{ Modality } & \multicolumn{2}{|c|}{ Number of patients } \\
\hline & & & Control & Optimisation \\
\hline A & 2 & $\begin{array}{l}\text { Siemens } \\
\text { Somtom }\end{array}$ & 20 & 14 \\
\hline B & 4 & $\begin{array}{l}\text { Siemens } \\
\text { Sensation }\end{array}$ & 20 & 12 \\
\hline C & 16 & $\begin{array}{l}\text { Siemens } \\
\text { Sensation }\end{array}$ & 20 & 12 \\
\hline $\mathrm{D}$ & 64 & \begin{tabular}{|l|} 
Toshiba \\
Aquilion
\end{tabular} & 20 & 10 \\
\hline
\end{tabular}

The evaluation of image quality was performed by four radiologists using a region-ofinterest (ROI) tool in the CT software. Moreover, evaluation of subjective image quality was performed by three expert radiologists from independent hospitals using a three-point Likert scale ( 3 , excellent; 2 , moderate; 1 , unacceptable). Inter-Rater reliability analysis was carried out to determine the degree of agreement among raters for subjective image quality scoring. Image quality evaluation was based on an assessment of the CT number, which expressed as Hounsfield units (HU). To better visualize changes in noise across the images, maps of the standard deviation (SD) for the CT number within the images were created. These maps were created by sectioning the axial CT images into ROIs and calculating the SD of the CT number for each ROI. The resulting images qualitatively show how the image noise varies.

Organ doses were determined by using VirtualDose software which designed as an application permits users to access its functions via the Internet (Virtual Phantoms, Inc., New York, USA). As reported by Ding et al. the VirtualDose software is extremely reliable and fully functional in reporting organ doses for the adult patient (19). The CTDIvol (in mGy) and DLP (in mGy.cm), indicated by the CT software, were recorded for each scanner to estimate the effective dose for patients. The effective dose for abdomen examinations was calculated based on $\mathrm{k}$ values reported by Shrimpton et al. $(20,21)$. The tissue weighting factors were obtained from the International Commission on Radiological Protection (ICRP) publication 103 (22) and ICRP publication 60 (23) based on Monte Carlo simulations.

The risk of developing cancer in a particular organ following CT abdomen after irradiation was estimated based on equation (1).

$$
\mathrm{CP}=\bar{H}_{T} \times f_{T}
$$

Where; CP is cancer probability, $\bar{H}_{T}$ is the mean organ equivalent dose and $f_{\mathrm{T}}$ is the risk coefficients reported by the ICRP publication 103 (22).

All statistics were calculated using either Microsoft Office Excel 2013 (Microsoft Inc, Redmond, WA) and/or the SPSS version 14 (SPSS Inc, Chicago, IL). A two-sample t-test was used to analyze the study results.

\section{RESULTS}

Demographic data (the age and gender) and scan parameters for the patients undergoing abdomen CT examinations before and after optimisation are presented in table 2 and 3 . The ratio between the number of males and females patients was estimated to be 1.37:1. The median patient weight and the body mass index (BMI) for all patients were ranged between $55-68 \mathrm{~kg}$ and $18-25 \mathrm{~kg} / \mathrm{m}^{2}$, respectively. It is worth mentioning that the median patient size was less than the standard sized person recommended by ICRP (23). The $\mathrm{kVp}, \mathrm{mAs}$, pitch, and slice thickness were constant in the four groups, whereas the gantry rotation time is reduced by 16.7 to $30 \%$ (table 3 ).

Table 4 presents the patient's dose results in terms of CTDIvol, DLP and effective dose. Among the four groups, the CT dose reduction of $30 \%$ was achieved by using optimisation technique. The results also show that the DLP and effective dose values decreased to $55 \%$ and $49 \%$, respectively. Regarding the relationship 
between CT doses of control and optimisation groups the t-test performed showed the group doses correlation were statistically insignificant $(\mathrm{P}<0.05)$.

Table 5 shows the results of image quality evaluation. As image quality evaluation in this study based on an assessment of the CT number, table 5 shows the range and average of CT number obtained, as well as the average of the standard deviation of the CT number for each ROI. In general, the assessment of the objective image quality showed that the gantry rotation time reduction results in insignificant image noise (table 5). The mean image noise in group A was outside the acceptable range. However, all
CT images of group B, C, and D were within the acceptable range and easy to diagnose. Furthermore, the rating of "excellent" was obtained by the three radiologists with an inter-rater reliability of $74 \%$ for the four groups.

Table 6 shows the organ equivalent doses used to estimate the CP risks for male and female patients. It is also reveals that the $\mathrm{CP}$ for different organs was in a magnitude of $10^{-6}$. The colon and stomach have the highest $\mathrm{CP}$, whereas the gonad has the lowest CP. The disparity between $\mathrm{CP}$ values can be explained due to the variation between the radiosensitivity of organs and the position of organs inside or outside the radiation field.

Table 2. Demographics information for the patients undergoing abdomen CT examinations before and after optimisation.

\begin{tabular}{|c|c|c|c|c|}
\hline \multirow{2}{*}{ Group Code } & \multirow{2}{*}{ Patients category } & Age(year)* & \multicolumn{2}{|c|}{ Gender } \\
\cline { 3 - 5 } & & & Male & Female \\
\hline \multirow{2}{*}{ A } & Control & $46.5 \pm 11.7(35-80)$ & 13 & 7 \\
\cline { 2 - 5 } & Optimisation & $39.6 \pm 16.9(20-74)$ & 9 & 5 \\
\hline \multirow{2}{*}{ B } & Control & $56.2 \pm 13.7(14-70)$ & 5 & 15 \\
\cline { 2 - 5 } & Optimisation & $56.5 \pm 13.3(30-70)$ & 6 & 6 \\
\hline \multirow{2}{*}{ C } & Control & $49.9 \pm 16.1(20-80)$ & 13 & 7 \\
\cline { 2 - 5 } & Optimisation & $43.4 \pm 8.3(35-62)$ & 9 & 3 \\
\hline \multirow{2}{*}{ D } & Control & $53.0 \pm 16.5(32-91)$ & 13 & 7 \\
\cline { 2 - 5 } & Optimisation & $48.3 \pm 17.7(28-82)$ & 6 & 4 \\
\hline
\end{tabular}

*mean of age values \pm standard deviation (minimum-maximum)

Table 3. Parameters of image acquisition before and after optimisation during abdomen CT examinations.

\begin{tabular}{|c|c|c|c|c|c|c|c|}
\hline Group Code & Patients category & $k V p$ & mAs & \begin{tabular}{|c|}
$\begin{array}{c}\text { Slice thickness } \\
(\mathrm{mm})\end{array}$ \\
\end{tabular} & Pitch & \begin{tabular}{|c|}
$\begin{array}{c}\text { Rotation Time } \\
(\mathrm{sec})\end{array}$ \\
\end{tabular} & $\begin{array}{c}\text { Reduction } \\
(\%)\end{array}$ \\
\hline \multirow{2}{*}{$A$} & Control & 110 & 120 & 5 & 2.00 & 1.0 & \multirow{2}{*}{30.0} \\
\hline & Optimisation & 110 & 120 & 5 & 2.00 & 0.7 & \\
\hline \multirow{2}{*}{ B } & Control & 120 & 200 & 5 & 4.50 & 0.7 & \multirow{2}{*}{28.6} \\
\hline & Optimisation & 120 & 200 & 5 & 4.50 & 0.5 & \\
\hline \multirow{2}{*}{$\mathrm{C}$} & Control & 120 & 160 & 5 & 0.84 & 0.7 & \multirow{2}{*}{28.6} \\
\hline & Optimisation & 120 & 160 & 5 & 0.84 & 0.5 & \\
\hline \multirow{2}{*}{ D } & Control & 120 & 150 & 5 & 1.48 & 0.6 & \multirow{2}{*}{16.7} \\
\hline & Optimisation & 120 & 150 & 5 & 1.48 & 0.5 & \\
\hline
\end{tabular}


Table 4. CT dose parameters.

\begin{tabular}{|c|c|c|c|c|}
\hline & Control & Optimisation & Mean dose reduction (\%) & $P$ value \\
\hline \multicolumn{5}{|c|}{ Group A } \\
\hline $\mathrm{CTDI}_{\mathrm{vol}}(\mathrm{mGy})^{*}$ & $5.5 \pm 1.1(2.8-6.2)$ & $4.3 \pm 1.1(2.2-6.2)$ & $26.4 \%$ & $<0.01$ \\
\hline DLP (mGy.cm) * & $222.2 \pm 57.5(105.0-312.0)$ & $173.9 \pm 54.3(82.0-276.0)$ & $24.4 \%$ & $<0.01$ \\
\hline Effective dose (mSv) & 3.3 & 2.6 & $21.2 \%$ & $<0.01$ \\
\hline \multicolumn{5}{|c|}{ Group B } \\
\hline $\mathrm{CTDI}_{\mathrm{vol}}(\mathrm{mGy})^{*}$ & $59.1 \pm 15.3(41.2-82.8)$ & $45.3 \pm 4.2(41.2-56.7)$ & $26.4 \%$ & $<0.01$ \\
\hline $\mathrm{DLP}$ (mGy.cm)* & $2906.6 \pm 674.8(1500.2-3709.1)$ & $1475.7 \pm 378.8(903.1-2119.1)$ & $55.4 \%$ & $<0.01$ \\
\hline Effective dose (mSv) & 43.5 & 22.1 & $49.2 \%$ & $<0.01$ \\
\hline \multicolumn{5}{|c|}{ Group C } \\
\hline $\mathrm{CTDI}_{\mathrm{vol}}(\mathrm{mGy})^{*}$ & $7.1 \pm 1.8(4.4-11.3)$ & $5.2 \pm 0.8(4.2-6.8)$ & $30.1 \%$ & $<0.01$ \\
\hline DLP (mGy.cm)* & $339.9 \pm 100.7(146.0-534.0)$ & $241.0 \pm 46.2(184.0-316.0)$ & $34.1 \%$ & $<0.01$ \\
\hline Effective dose (mSv) & 5.1 & 3.6 & $29.4 \%$ & $<0.01$ \\
\hline \multicolumn{5}{|c|}{ Group D } \\
\hline $\mathrm{CTDI}_{\mathrm{vol}}(\mathrm{mGy})^{*}$ & $21.9 \pm 0.0(21.9-21.9)$ & $19.7 \pm 1.8(18.3-21.9)$ & $10.6 \%$ & $<0.01$ \\
\hline $\mathrm{DLP}(\mathrm{mGy} . \mathrm{cm})^{*}$ & $938.5 \pm 200.0(629.0-1297.0)$ & $797.8 \pm 135.4(623.7-973.5)$ & $16.2 \%$ & $<0.01$ \\
\hline Effective dose (mSv) & 14.1 & 11.9 & $15.6 \%$ & $<0.01$ \\
\hline
\end{tabular}

Table 5. Comparison between image noise differences (Diff) in the four CT scanners before and after optimisation for abdomen protocol.

\begin{tabular}{|c|c|c|c|}
\hline Group Code & Patients category & Image noise (HU)* & Diff between control and optimization (\%) \\
\hline \multirow{2}{*}{ A } & Control & $13.4 \pm 3.3(8.0-23.0)$ & \multirow{2}{*}{10.5} \\
\hline & Optimisation & $14.8 \pm 3.4(9.6-21.1)$ & \\
\hline \multirow{2}{*}{ B } & Control & $7.8 \pm 1.7(4.4-9.7)$ & \multirow{2}{*}{5.1} \\
\hline & Optimisation & $8.2 \pm 2.6(3.9-13.0)$ & \\
\hline \multirow{2}{*}{ C } & Control & $11.5 \pm 3.1(5.3-16.3)$ & \multirow{2}{*}{0.9} \\
\hline & Optimisation & $11.6 \pm 4.0(5.4-18.6)$ & \\
\hline \multirow{2}{*}{ D } & Control & $7.2 \pm 1.3(4.4-9.6)$ & \multirow{2}{*}{4.6} \\
\hline & Optimisation & $7.5 \pm 1.2(6.0-10.3)$ & \\
\hline
\end{tabular}

Table 6. CP for male and female patients undergoing abdomen CT examinations.

\begin{tabular}{|c|c|c|c|c|c|c|}
\hline & \multicolumn{7}{|c|}{ Organ equivalent dose (mSv) } \\
\cline { 2 - 7 } & Colon & Bladder & Gonads & Liver & Bone Marrow & Stomach \\
\hline Male risk factor $\times 10^{-4}\left(\mathrm{~Sv}^{-1}\right)$ & 73 & 40 & 12 & 31 & 24 & 51 \\
\hline Female risk factor $\times 10^{-4}\left(\mathrm{~Sv}^{-1}\right)$ & 33 & 39 & 12 & 16 & 22 & 70 \\
\hline \multicolumn{7}{|c|}{ Group A } \\
\hline Male & 3.02 & 3.37 & 0.12 & 5.39 & 0.55 & 3.59 \\
\hline Female & 3.39 & 3.44 & 0.23 & 5.61 & 0.63 & 3.89 \\
\hline CP for Male $\times 10^{-6}$ & 22.05 & 13.48 & 0.14 & 16.71 & 1.32 & 18.31 \\
\hline CP for Female $\times 10^{-6}$ & 11.19 & 13.42 & 0.28 & 8.98 & 1.39 & 27.23 \\
\hline \multicolumn{7}{|c|}{ Group B } \\
\hline Male & 29.75 & 24.89 & 1.10 & 43.73 & 5.13 & 28.38 \\
\hline Female & 32.90 & 25.65 & 2.78 & 44.68 & 6.13 & 30.49 \\
\hline CP for Male $\times 10^{-6}$ & 217.18 & 99.56 & 1.32 & 135.56 & 12.31 & 144.74 \\
\hline CP for Female $\times 10^{-6}$ & 108.60 & 100.04 & 3.34 & 71.49 & 13.49 & 213.43 \\
\hline \multicolumn{7}{|c|}{ Group C } \\
\hline Male & 3.41 & 2.85 & 0.13 & 5.01 & 0.59 & 3.25 \\
\hline Female & 3.04 & 2.23 & 0.28 & 4.37 & 0.63 & 2.67 \\
\hline CP for Male $\times 10^{-6}$ & 24.89 & 11.40 & 0.16 & 15.53 & 14.16 & 16.58 \\
\hline CP for Female $\times 10^{-6}$ & 10.03 & 8.70 & 0.34 & 6.99 & 1.39 & 43.55 \\
\hline \multicolumn{7}{|c|}{ Group D } \\
\hline Male & 12.94 & 10.82 & 0.48 & 19.02 & 2.23 & 12.34 \\
\hline FP for Male $\times 10^{-6}$ & 14.31 & 11.16 & 1.21 & 19.43 & 2.67 & 13.26 \\
\hline CP for Female $\times 10^{-6}$ & 94.46 & 43.28 & 0.58 & 58.96 & 5.35 & 62.93 \\
\hline
\end{tabular}

Int. J. Radiat. Res., Vol. 19 No. 1, January 2021 


\section{DISCUSSION}

Several researchers reported that accelerating CT gantry rotation and increasing the width of the detector improves performance and making CT modality more appropriate against artefacts or other image quality components $(24,25)$. However, the image noise and artefacts in CT imaging are still one of the common significant issues. Furthermore, some clinical cases, accelerating gantry rotation can cause supplementary artefacts and noise. For that purpose, we evaluated the influence of decreasing rotation time on radiation exposure and imaging quality for the patients undergoing abdomen examinations in several CT scanners.

The CT dose depends on the patients' size and scan parameters. No significant difference was observed in terms of height, weight, and BMI between the two patient categories. Hence, the comparison between the optimisation and control categories will be more reliable. As previously mentioned in the results section, a reduction of CTDIvol and DLP of up to $30 \%$ and $55 \%$ of the total dose were achieved, respectively. Besides, the mean CTDIvol was 5.5 mGy and DLP was 222.2 mGy.cm for Group A and CTDIvol was 59.1 mGy and DLP was 2906.6 mGy $\mathrm{cm}$ for Group B (table 4). The disparity in the range of CTDIvol and DLP values between the four patients' groups can be due to variation in the pitch and mAs used in the CT scanners. Additional factors such as insufficient skills of the technologists and practitioners in the newly emerging technology were also reported in the literature (26).

Most of the CT images obtained in this study were acceptable and easy to diagnose. It is important to underline that the image noise measured for group B, C, and D are within the acceptable limits as recommended by the Institute of Physics and Engineering in Medicine (IPEM) (27). However, the results of group A showed that faster gantry rotation time increases image noise. By excluding group $\mathrm{A}$, the degree of agreement on image quality among radiologists shows that the gantry rotation time has only a small impact on overall image quality. Present results are similar to Klink et al. study in that rotation time have a limited influence on the quality of images (28). It is worth mentioning that the unacceptable range of noise obtained in group $\mathrm{A}$, demonstrate that the accelerating time of gantry rotation does not always maintain the best image noise.

The results of this study revealed that the mean DLP in group B for abdomen examinations, before and after the optimisation, is higher compared with previously published studies (4, 29-31). Conversely, the DLP values of groups $\mathrm{A}$ and $\mathrm{C}$ are lower than the others (figure 1). The possible explanation of the higher DLP values for group $B$ is the variation of exposure parameter, sample size or patient weight. For example, the $\mathrm{kVp}$ and $\mathrm{mAs}$ values of all CT scanners used in this study have remained constant in the optimisation category. It was possible to reduce the tube voltage also in this study, as the use of low tube voltage settings has many benefits such as radiation dose reduction and higher contrast enhancement (31, 32). However, the reduction of the $\mathrm{kVp}$ and $\mathrm{mAs}$ reduction, as well as decreasing rotation time, can be compromising the diagnostic findings.

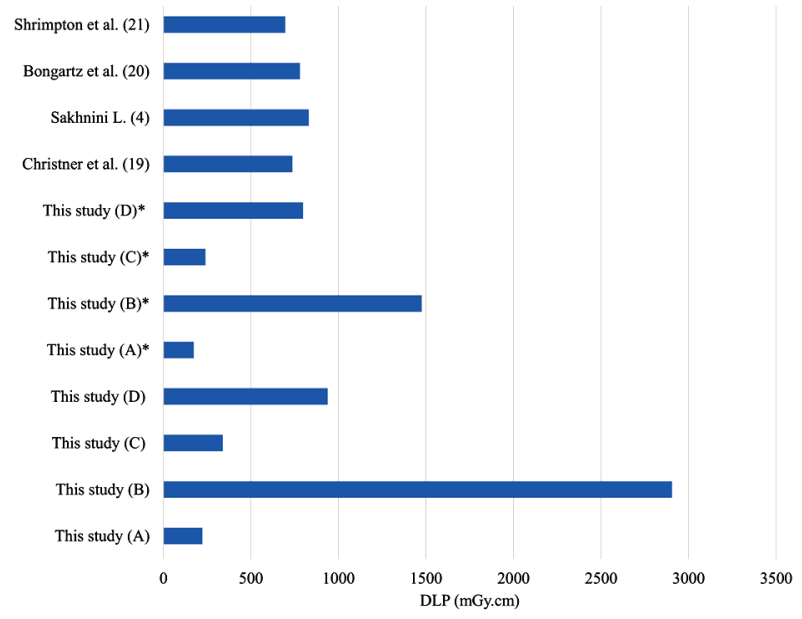

Figure 1. Comparison of abdominal CT Dose values with DRLS reported in previous studies (4,19-21). *DLP after optimisation.

CT abdomen involves direct irradiation of the colon, liver and stomach, which necessitates evaluating the dose received by scattered radiation. In this study, additional organs located outside the radiation field, such as bladder and gonads, were included. In general, 
we found that the effective dose in groups A and $D$ for females is higher than males with a factor of 1.1. However, no direct correlation was found between the CT scanners types and genders. The organ equivalent doses presented in table 6 shows that the liver has the highest organ dose in both males and females, whereas the CP for stomach and colon is highest compared to other organs in all groups. The colon, liver, and stomach in both genders have the highest CP comparing to the gonads, bladder and bone morrow. Further, the cancer risk of the colon in males was found higher than females and this can be attributed to the difference in the radiosensitivity of the colon in both genders. Therefore, abdomen CT procedure for both genders needs to be cautiously justified in view of high liver dose and because of the high CP incidence for stomach and colon. On the other hand, the liver and stomach dose values were ranged between 5.39- $43.73 \mathrm{mSv}$ and 3.59-30.49 $\mathrm{mSv}$, respectively (table 6). The main reason for higher doses is different mAs values in this study. The maximum stomach, colon and liver doses reported in this study compared with a previous study were varied by factor up to 1.1 $(34,35)$. Thus, the convergence between the results of the maximum organ effective doses in this study and the mean results of previous studies enhances the possibility of dose optimisation through increasing the speed of gantry rotation.

The overall cancer risk after optimisation was reduced with $21 \%, 49 \%, 29 \%$, and $16 \%$, for group A, B, C, and D respectively. Consequently, the attention of all technologists necessary to take advantage of the latest dose reduction methods, and simultaneously the radiologists should commitment to the clinical advantages and justify any decision that alters the situation of radiation exposure of the patient undergoing CT scan. The dose reduction of up to $30 \%$ was achieved without the loss of diagnostic accuracy for most of the patients $(70 \%)$ in the optimisation groups. The major limitation of this study was the relatively small number of patients included and the size of the patient, which could potentially influence the dose reduction.

Int. J. Radiat. Res., Vol. 19 No. 1, January 2021

\section{CONCLUSION}

To the best of our knowledge, this work is the first to study the influences of accelerating gantry rotation time on dose reduction and image quality in abdominal CT imaging. By lowering gantry rotation times, a radiation dose reduction of up to $30 \%$ was achieved for 4, 16 and 64 slice CT scanners without compromising the diagnostic findings. However, faster gantry rotation time can increase image noise for 2-slice CT scanners. Dose reduction requires continuous efforts and close cooperation between technicians, radiologists and regulatory bodies. Finally, the optimisation protocols must be applied with care to guarantee that they are tailored to patient size and clinical need.

\section{ACKNOWLEDGEMENT}

The authors would like to thank the co-operation of radiologists and technologists of the Najran University, Princess Nora bint Abdul Rahman University, and Prince Sattam bin Abdulaziz University participating in this study. This study was funded by Scientific Research Deanship of Najran University (Grant No. NU/ MID/16/009).

\section{Conflicts of interest: Declared none.}

\section{REFERENCES}

1. United Nations Scientific Committee on the Effects of the Atomic Radiation UNSCEAR (2000) Medical Radiation Exposures. Annex D, UNSCEAR report to the general assembly, New York.

2. National Council on Radiation Protection and Measurements NCRP (2009) Ionizing Radiation Exposure of the Population of the United States. NCRP Report No. 160, Bethesda, USA.

3. American Association of Physicists in Medicine AAPM (2008) The Measurement, reporting, and management of radiation dose in CT, Report of AAPM Task group 23 of the Diagnostic Imaging Council CT Committee. AAPM Report No 96.

4. Sakhnini $L$ (2017) CT radiation dose optimisation and reduction for routine head, chest and abdominal CT exami- 
nation. Radiol Diagn Imag, 2(1):1-4.

5. European Commission EC (1999) European Guidelines on Quality Criteria for Computed Tomography. Report EUR $16262 \mathrm{EN}$, Brussels, Belgium.

6. McNitt-Gray MF (2002) AAPM/RSNA Physics Tutorial for Residents: Topics in CT. Radiation dose in CT. Radiographics, 22(6):1541-1553.

7. International Commission on Radiological Protection (2000) Managing Patient Dose in Computed Tomography. ICRP Publication 87. Ann. ICRP 30(4).

8. Kalra MK, Dang P, Singh S, Saini S, Shepard JA (2009) Inplane shielding for $\mathrm{CT}$ : effect of off-centering, automatic exposure control and shield-to-surface distance. Korean J Radiol, 10:156-163.

9. Rehani MM and Berry M (2000) Radiation doses in computed tomography: the increasing doses of radiation need to be controlled. BMJ, 320: 593-594.

10. Kalra MK, Maher MM, Toth TL, Hamberg LM, Blake MA, Shepard JA, Saini S (2004) Strategies for CT radiation dose optimization. Radiology, 230: 619-628.

11. Saravanakumar A, Vaideki K, Govindarajan KN, Devanand B, Jayakumar S, Sharma SD (2016) Establishment of CT diagnostic reference levels in select procedures in South India. Int J Radiat Res, 14(4):341-347.

12. Ay MR, Shahriari M, Sarkar S, Ghafarian P (2004) Measurement of organ dose in abdomen-pelvis $C T$ exam as a function of $\mathrm{mA}, \mathrm{KV}$ and scanner type by Monte Carlo method. Int J Radiat Res, 1(4):187-194.

13. Ghavami SM, Mesbahi A, Pesianian I (2012) Patient doses from $X$-ray computed tomography examinations by a single-array detector unit: Axial versus spiral mode. Int J Radiat Res, 10(2):89-94.

14. Sadri L, Khosravi HR, Setayeshi S (2013) Assessment and evaluation of patient doses in adult common CT examinations towards establishing national diagnostic reference levels. Int J Radiat Res, 11(4): 245-252.

15. National Radiological Protection Board (1994) Estimation of Effective Dose in Diagnostic Radiology from Entrance Surface Dose and Dose-Area Product Measurements. NRPB Report No. 262.

16. Diagnostic and Interventional cardiology (2013) Canon Medical System, USA. https://www.dicardiology.com/ product/toshiba-releases-640-slice-ct-scanner

17. Thakur Y, McLaughlin PD, Mayo JR (2013) Strategies for radiation dose optimization. Curr Radiol Rep, 1: 1-10.

18. Beeres M, Wichmann JL, Paul J, Mbalisike E, Elsabaie M, Vogl TJ, Nour-Eldin NE (2015) CT chest and gantry rotation time: does the rotation time influence image quality? Acta Radiol, 56(8):950-954.

19. Ding A, Gao Y, Liu H, Caracappa PF, Long DJ, Bolch WE, Liu B, Xu XG (2015) VirtualDose: a software for reporting organ doses from CT for adult and pediatric patients. Phys Med Biol, 60: 5601-5625.

20. Shrimpton P (2004) Assessment of patient dose in CT. In: EUR. European guidelines for multislice computed tomography funded by the European Commission 2004: contract number FIGMCT2000-20078-CTTIP. Luxembourg: European Commission: Appendix C.

21. Shrimpton PC, Hillier MC, Lewis MA, Dunn M (2003) Doses from computed tomography (CT) examinations in the UK: 2003 review. Chilton, UK: National Radiologic.

22. International Commission on Radiological Protection ICRP (2007) The 2007 Recommendations of the International Commission on Radiological Protection. Annals of the ICRP 37, ICRP Publication 103, Pergamon Press, Oxford.

23. International Commission on Radiological Protection ICRP (1990) Recommendations of the International Commission on Radiological Protection. Annals of the ICRP 1991, ICRP Publication 60, Pergamon Press, Oxford.

24. Kroft LJ, Roelofs JJ, Geleijns J (2010) Scan time and patient dose for thoracic imaging in neonates and small children using axial volumetric 320-detector row CT compared to helical 64-, 32-, and 16- detector row CT acquisitions. Pediatr Radiol, 40: 294-300.

25. Hinkmann FM, Voit HL, Anders K, Baum U, Seidensticker P, et al. (2009) Ultra-fast carotid CT-angiography: low versus standard volume contrast material protocol for a 128-slice CT-system. Invest Radiol, 44: 257-264.

26. Paterson A and Frush DP (2007) Dose reduction in paediatric MDCT: general principles. Clin Radiol 62(6): 507-517.

27. Institute of Physics and Engineering in Medicine IPEM (2005) Recommended standards for the routine performance testing of diagnostic X-ray imaging systems. IPEM Report No. 91.

28. Klink T, Regier M, Stevendaal Uv, Grass M, et al. (2012) Accelerating image acquisition in 64-MDCT: the influence of scan parameters on image resolution and quality in a phantom study. Clin Imaging, 36: 334-344.

29. Christner JA, Kofler JM, McCollough CH (2010) Estimating effective dose for $\mathrm{CT}$ using dose-length product compared with using organ doses: Consequences of adopting international commission on radiological protection publication 103 or dual-energy scanning. AJR. Am J Roentgenol, 194 (4): 881-9.

30. Bongartz G, Golding SJ, Jurik AG, Leonardi M, et al. (2004) Quality criteria for multislice computed tomography. Results from a European concerted action on CT (FIGM-CT2000-20078) Appendix B: European field survey on MSCT.

31. Shrimpton PC, Hillier MC, Lewis MA, Dunn M (2006) National survey of doses from CT in the UK: 2003. Br J Radiol, 79: 968-980.

32. Guimarães LS, Fletcher JG, Harmsen WS, et al. (2010) Appropriate patient selection at abdominal dual-energy CT using $80 \mathrm{kV}$ : relationship between patient size, image noise, and image quality. Radiology, 257(3): 732-742.

33. Seyal AR, Arslanoglu A, Abboud SF, Sahin A, Horowitz JM, Yaghmai V (2015) CT of the Abdomen with Reduced Tube Voltage in Adults: A Practical Approach. Radiographics, 35 (7): 1922-1939.

34. Ngaile JE and Msaki PK (2006) Estimation of patient organ doses from CT examinations in Tanzania. J Appl Clin Med Phys, 7(3): 80-94.

35. Griglock TM, Sinclair L, Mench A, Cormack B, Bidari S, Rill L, Arreola M (2015) Determining Organ Doses from CT with Direct Measurements in Postmortem Subjects: Part 1-Methodology and Validation. Radiology, 277(2): 463470.

Int. J. Radiat. Res., Vol. 19 No. 1, January 2021 